\title{
Convergence Guarantees for Discrete Mode Approximations to Non-Markovian Quantum Baths
}

\author{
Rahul Trivedi," Daniel Malz@, and J. Ignacio Cirac® \\ Max-Planck-Institut für Quantenoptik, Hans-Kopfermann-Straße 1, 85748 Garching, Germany \\ and Munich Center for Quantum Science and Technology (MCQST), Schellingstraße 4, D-80799 Munich, Germany
}

(Received 8 September 2021; revised 8 November 2021; accepted 12 November 2021; published 15 December 2021)

\begin{abstract}
Non-Markovian effects are important in modeling the behavior of open quantum systems arising in solid-state physics, quantum optics as well as in study of biological and chemical systems. The nonMarkovian environment is often approximated by discrete bosonic modes, thus mapping it to a Lindbladian or Hamiltonian simulation problem. While systematic constructions of such modes have been previously proposed, the resulting approximation lacks rigorous and general convergence guarantees. In this Letter, we show that under some physically motivated assumptions on the system-environment interaction, the finitetime dynamics of the non-Markovian open quantum system computed with a sufficiently large number of modes is guaranteed to converge to the true result. Furthermore, we show that this approximation error typically falls off polynomially with the number of modes. Our results lend rigor to classical and quantum algorithms for approximating non-Markovian dynamics.
\end{abstract}

DOI: 10.1103/PhysRevLett.127.250404

Quantum systems invariably interact with their environment, and any simulation technique used to model their behavior needs to capture this interaction. Traditionally, such interactions are analyzed within the Markovian approximation, wherein the system dynamics is described by the Lindbladian master equation [1]. However, a number of quantum systems arising in solid-state physics [2-5], quantum optics [6-10], as well as quantum biology and chemistry [11-14] cannot be modeled accurately within the Markovian approximation and the non-Markovian nature of the environment needs to be explicitly taken into account.

Simulating non-Markovian open quantum systems is difficult since it is usually not possible to formulate a dynamical equation for the system state from a given physical model of the system-environment interaction. While it is generically expected that non-Markovian open quantum systems satisfy a master equation of the Nakajima-Zwanzig form $[13,15]$, or the time-convolutionless form [16-18], it is usually hard to obtain an explicit form of such a master equation except when the system is only weakly coupled to its environment [19-22]. Even though significant progress has been made in utilizing influence functionals or their variants for describing and

Published by the American Physical Society under the terms of the Creative Commons Attribution 4.0 International license. Further distribution of this work must maintain attribution to the author(s) and the published article's title, journal citation, and DOI. Open access publication funded by the Max Planck Society. simulating non-Markovian dynamics [23-31], the worstcase classical complexity of these approaches increases exponentially with time. An alternative approach is to identify and track a set of discrete modes that approximate the environment [32-34]. This maps the simulation of the non-Markovian open quantum system to a larger Hamiltonian or Lindbladian simulation problem, which can be solved using standard classical [35-39] or quantum algorithms [40-45].

For Gaussian bosonic environments [46,47], there are two prominent approaches to identifying these discrete modes. The first is to use the Lorentzian pseudomode theory [48-53], wherein the spectral density function of the non-Markovian environment is approximated by a finite sum of Lorentzians, each of which corresponds to an individual bosonic mode coupled to Markovian reservoir. The second method is to use star-to-chain transformation $[33,34]$, which uses the Lanczos iteration to identify a 1D chain of discrete bosonic modes with nearest neighbor couplings that approximate the environment and map the problem of computing non-Markovian quantum dynamics to a Hamiltonian simulation problem. While a systematic construction of these approximations has been given, their convergence properties are less well understood. For instance, classes of models where the Lorentzian pseudomode description is exact are known [48,49], but it is unknown if it can efficiently approximate a non-Markovian environment. More attention has been paid toward investigating the convergence properties of the star-to-chain transformation-convergence guarantees have been provided for models with a finite Lieb-Robinson velocity $[54,55]$, or for bounded memory kernels [56] that can 
possibly have an infinite Lieb-Robinson velocity [57]. However, these analyses do not extend to models with distributional (and hence unbounded) memory kernels, such as those commonly encountered in non-Markovian quantum optical systems [6,58].

In this Letter, we provide general and rigorous convergence guarantees for discrete mode approximations of non-Markovian Gaussian bosonic environments. We show that for a wide and physically motivated class of nonMarkovian models, both the Lorentzian pseudomode approximation and the star-to-chain transformation is guaranteed to converge and the approximation error falls off polynomially with the number of pseudomodes. Our results not only provide the first set of rigorous convergence guarantees for the pseudomode approximation, but also extend the convergence guarantees for the star-to-chain transformation to non-Markovian systems described by a distributional memory kernel, such as those commonly encountered in quantum optics $[6,58]$.

In order to perform this convergence study, there are several theoretical challenges that our work resolves. One of the key issues with analyzing models of non-Markovian environments is that the environment can support arbitrarily large energies and the quantum system often has nonvanishing couplings with high energy environment modes $[6,58]$. We identify a set of physically motivated sufficient mathematical conditions on the system-environment dynamics that allow for rigorously neglecting the high energy modes in the environment while studying finitetime dynamics. While this straightforwardly follows for problems where the system-environment couplings vanish at high energies, our analysis also includes distributional memory kernels. Combining this with an analysis of the Lorentzian pseudomode approximation and the starto-chain transformation within a truncated environmentenergy window, we provide convergence guarantees as well estimates of the rate of convergence for both of these methods.

We consider an open quantum system model, with a $d$-dimensional quantum system with Hilbert space $\mathcal{H}_{S}=\mathbb{C}^{d}$ (referred to as a "local system") interacting with a Gaussian environment whose Hilbert space, $\mathcal{H}_{E}$ is assumed to be a Fock space over $L^{2}(\mathbb{R})$. We denote by $a_{\omega}$ the annihilation operator for this Fock space and consider Hamiltonians of the form

$$
\begin{aligned}
H(t)= & H_{S}(t)+\int_{-\infty}^{\infty} \omega a_{\omega}^{\dagger} a_{\omega} d \omega \\
& +\int_{-\infty}^{\infty}\left[v(\omega) a_{\omega} L^{\dagger}+v^{*}(\omega) a_{\omega}^{\dagger} L\right] d \omega,
\end{aligned}
$$

where $L: \mathcal{H}_{S} \rightarrow \mathcal{H}_{S}$ is the operator describing the coupling of the system with the environment, and $v$ is the frequency dependent coupling function between the environment and the system. We point out that the Hamiltonian in Eq. (1) is only a provably (essentially) self-adjoint operator if $v \in$ $\mathrm{L}^{2}(\mathbb{R})$ [59]. However, several problems in quantum optics (e.g., systems with point coupling) are described by $v \in$ $C_{b}^{\infty}(\mathbb{R}) \cap \mathcal{S}^{\prime}(\mathbb{R})[60]$ that are tempered distributions and still result in well defined local system dynamics. Throughout this Letter, we will be interested in approximating the dynamics of the reduced state of the local system-for simplicity, we restrict ourselves to the case where the environment is initially in a vacuum state, extensions of the main results of this Letter to initially excited environment states is provided in Supplemental Material [61].

First, we outline two physically motivated assumptions that we make on the model under consideration that are sufficient conditions to allow us to neglect large environment frequencies. The first assumption makes mathematically precise the expectation that the effective timedomain kernel $K_{v}$ corresponding to the coupling function $v \in C_{b}^{\infty}(\mathbb{R}) \cap \mathcal{S}^{\prime}(\mathbb{R})$, defined by

$$
K_{v}(t)=\int_{-\infty}^{\infty}|v(\omega)|^{2} e^{-i \omega t} d \omega,
$$

is approximable by its restriction to a finite frequency window. Since $K$ can in general be a distribution, we first introduce a distributional quasinorm to quantify this approximation error.

Definition 1.-Let $v \in \mathbb{C}_{b}^{\infty}(\mathbb{R})$, and let $K_{v}$ be its corresponding kernel, then for $t>0$, define a quasinorm $\ell\left(K_{v}, t\right)$ of $K_{v}$ via [65]

$$
\begin{aligned}
\ell\left(K_{v}, t\right)= & \sup _{\substack{f \in A C_{\text {sym }}^{\geq 0}\left([0, t]^{2}\right) \\
f \neq 0}} \frac{1}{\|f\|_{[0, t]^{2}}^{\mathcal{S}}} \\
& \times \int_{s_{1}, s_{2}=0}^{t} K_{v}\left(t-s_{1}\right) K_{v}^{*}\left(t-s_{2}\right) f\left(s_{1}, s_{2}\right) d s_{1} d s_{2} .
\end{aligned}
$$

We note that our definition of this quasinorm involves two applications of the kernel on a test function, which itself is a function of two time indices - this choice of the quasinorm is natural since we are interested in quantifying the backaction of particles emitted into the environment on the local system dynamics, with there being two time indices needed to describe the reduced state of each particle in the environment.

Assumption 1.-The coupling function $v \in \mathbb{C}_{b}^{\infty}(\mathbb{R})$ is such that there is a function $V\left(\omega_{c}, t\right)$ which vanishes as $\omega_{c} \rightarrow \infty \forall t \geq 0$ and

$$
\ell\left(K_{v}-K_{v_{\omega_{c}}}, t\right) \leq V\left(\omega_{c}, t\right),
$$

where $v_{\omega_{c}}(\omega)=v(\omega)$ if $|\omega| \leq \omega_{c}$ and otherwise 0 .

By ensuring that the kernel is distributionally approximable within a finite frequency window, Assumption 1 ensures that the model described by Eq. (1) does not suffer 
from an ultraviolet divergence arising due to the environment being able to support arbitrarily high frequencies [66]. A number of models commonly considered in practice do satisfy this assumption: (i) Environments with squareintegrable coupling functions, i.e., $v \in C_{b}^{\infty}(\mathbb{R}) \cap L^{2}(\mathbb{R})$ satisfy Assumption 1 (Proposition 1 in Supplemental Material [61]). Physically important examples of such environments include environments with a Lorentzian coupling function which is typically used to model an atomic system interacting with an optical cavity [67]. (ii) Markovian environments, which correspond to a frequency independent coupling function $v(\omega)=v_{0}$ [58], also satisfy Assumption 1 (Proposition 2 in Supplemental Material [61]). (iii) Environments modeling retardation effects, described by coupling functions of the form $v(\omega)=$ $\sum_{i=1}^{M} v_{i} e^{i \omega \tau_{i}}$ are commonly used to model retardation effects [68]. These environments also satisfy the conditions of Assumption 1 (Proposition 2 in Supplemental Material [61]). We point out that while the kernel might be approximable within a finite frequency window, due to the infinitedimensional nature of the environment's Hilbert space, it does not immediately follow that the reduced state of the local system is also similarly approximable.

To proceed further, we introduce a second assumption that ensures physically meaningful joint systemenvironment dynamics. To state this assumption mathematically, we introduce the $N$-point Green's function of the localized system.

Definition 2 (N-point Green's function). $-\forall s \in[0, t]^{N}$, $G_{N}(s ; t) \in \mathfrak{L}\left(\mathcal{H}_{S}\right)$ [69] via

$$
G_{N}(s ; t)=\left\langle\operatorname{vac}\left|U(t, 0) \mathcal{T}\left[\prod_{i=1}^{N} L\left(s_{i}\right)\right]\right| \operatorname{vac}\right\rangle,
$$

where $U\left(\tau_{1}, \tau_{2}\right)$ is the propagator corresponding to the Hamiltonian in Eq. (1) and $L(\tau)=U(0, \tau) L U(\tau, 0)$ is the operator $L$ in the Heisenberg picture.

The physical significance of the $N$-point Green's function is that it determines the projection of the environment state on the $N$-particle subspace, as is made explicit in the following lemma (proved in Supplemental Material [61]).

Lemma 1.-Let $|\psi(t)\rangle=U(t, 0)(|\sigma\rangle \otimes \mid$ vac $\rangle)$, where $|\sigma\rangle$ is a system state, then

$$
|\psi(t)\rangle=\sum_{N=0}^{\infty}\left|\psi^{N}(t)\right\rangle,
$$

where

$$
\left|\psi^{N}(t)\right\rangle=\frac{1}{N !} \int_{\omega \in \mathbb{R}} F_{N}(\omega ; t)\left[\prod_{i=1}^{N} v^{*}\left(\omega_{i}\right) a_{\omega_{i}}^{\dagger}\right]|\sigma\rangle \otimes|\mathrm{vac}\rangle d \omega,
$$

and $\forall \omega \in \mathbb{R}^{N}$,

$$
F_{N}(\omega ; t)=\int_{s \in[0, t]^{N}} G_{N}(s ; t) e^{-i\langle\omega,(t-s)\rangle} d s .
$$

Furthermore, as is made precise in Lemma 2 (proved in Supplemental Material [61]), the $N$-point Green's function is bounded, and consequently the norm of the $N$-particle projection of the environment state is also bounded.

Lemma 2.-If $v \in \mathbb{C}_{b}^{\infty}(\mathbb{R})$, then

$$
\left\|G_{N}(s, t)\right\| \leq\|L\|^{N} \quad \forall N \in \mathbb{N}, \quad t \geq 0, \quad s \in[0, t]^{N},
$$

and

$$
\|\left|\psi^{N}(t)\right\rangle \|^{2} \leq \frac{\|v\|_{\infty}^{2 N}\|L\|^{2 N}(2 \pi t)^{N}}{N !} \quad \forall N \in \mathbb{N}, \quad t \geq 0 .
$$

Our second assumption can be interpreted as a bound on the rate at which the local system can emit or absorb an excitation from the environment. Any physically reasonable model of the environment, despite its non-negligible couplings with high frequency environment modes, is expected to satisfy this assumption.

Assumption 2. $\forall t \geq 0, \quad s \in[0, t]^{N-1}$, the map $G_{N}(\{\cdot, s\} ; t):[0, t] \rightarrow \mathfrak{L}\left(\mathcal{H}_{S}\right)$ is absolutely continuous and $\exists \gamma(t)>0$ such that

$$
\underset{\left\{s_{0}, s\right\} \in[0, t]^{N}}{\operatorname{esssup}}\left\|\partial_{s_{0}} G_{N}\left(\left\{s_{0}, s\right\} ; t\right)\right\| \leq \gamma(t)\|L\|^{N} .
$$

This assumption can be proved for two cases: (1) Markovian environments, i.e., environments with a frequency independent coupling constant $\left[v(\omega)=v_{0}\right]$. In this case, an application of the quantum regression theorem can be used to show that Assumption 2 is satisfied for such environments (Proposition 3 in Supplemental Material [61]). (2) Environments with a square integrable coupling constant, Assumption 2 can again be rigorously proven (Proposition 4 in Supplemental Material [61]).

With these two assumptions, we can now prove the convergence of the pseudomode theory [48] and star-tochain transformation [33] for simulating non-Markovian quantum systems. Our first result, proved in Supplemental Material [61], rigorously shows that in a finite amount of time, the localized system cannot excite arbitrarily high frequencies in the environment, and the environment can thus be approximated within a finite energy window. More precisely,

Theorem 1.-Suppose $v \in C_{b}^{\infty}(\mathbb{R}) \cap \mathcal{S}^{\prime}(\mathbb{R})$ is a coupling function such that Assumptions 1 and 2 are satisfied. Denoting by $\rho(t)$ the reduced density matrix of the local system at time $t$ when an initial state $|\sigma\rangle \otimes|\mathrm{vac}\rangle$ is evolved under the Hamiltonian in Eq. (1) and by $\rho_{\omega_{c}}(t)$ the reduced density matrix of the local system at time $t$ when the same initial state is evolved under the Hamiltonian

$$
\begin{aligned}
H_{\omega_{c}}(t)= & H_{S}(t)+\int_{-\infty}^{\infty} \omega a_{\omega}^{\dagger} a_{\omega} d \omega \\
& +\int_{-\omega_{c}}^{\omega_{c}}\left(v(\omega) a_{\omega} L^{\dagger}+v^{*}(\omega) a_{\omega}^{\dagger} L\right) d \omega,
\end{aligned}
$$




$$
\left\|\rho(t)-\rho_{\omega_{c}}(t)\right\|_{\mathrm{tr}} \leq \varepsilon\left(\omega_{c}, t\right),
$$

where $\varepsilon\left(\omega_{c}, t\right)$ is the cutoff error given by

$$
\varepsilon\left(\omega_{c}, t\right)=\frac{f_{1}(t)}{\sqrt{\omega_{c}}}+\int_{0}^{t} f_{2}(\tau) \sqrt{V\left(\omega_{c}, \tau\right)} d \tau .
$$

Here $V\left(\omega_{c}, t\right)$ is defined in Assumption 1 and

$$
\begin{aligned}
& f_{1}(\tau)=\sqrt{2}\|v\|_{\infty}\|L\|(2+\gamma(\tau) \tau) e^{\pi\|v\|_{\infty}^{2}\|L\|^{2} \tau}, \\
& f_{2}(\tau)=\sqrt{2}\|L\|^{2} \tau(1+\gamma(\tau) \tau) e^{\pi\|v\|_{\infty}^{2}\|L\|^{2} \tau},
\end{aligned}
$$

where $\gamma(t)$ is introduced in Assumption 2.

The dependence of the cutoff error on $\omega_{c}$ is a sum of two terms - one that falls off as $O\left(1 / \sqrt{\omega_{c}}\right)$, which can be interpreted as the consequence of introducing a rectangular frequency window on the emitted photon wave packet, and a second term which depends on the error introduced in approximating the time-domain kernel $K_{v}$ within a frequency window (Assumption 1). The magnitude of this error, as given by the functions $f_{1}, f_{2}$, depends on the strength of the coupling between the system and environment $\left(\propto\|v\|_{\infty},\|L\|\right)$, as well as on the rate at which particles are exchanged with the environment $[\gamma(t)$ introduced in Assumption 2]. The result of Theorem 1 is thus in line with our intuition that a larger cutoff frequency is needed for systems which strongly couple to the environment, and absorb or emit particles very rapidly from the environment. Furthermore, the error grows exponentially with time $t$-this is a consequence of the fact that the local system can in principle emit an arbitrarily large number of particles into the environment while only being constrained by the bound in Lemma 2. In practice, we expect the errors grow only polynomially with $t$, with the polynomial to depend on the maximum number of particles that the local system can emit into the environment.

Next, we consider the pseudomode method [48], which approximates the non-Markovian dynamics of the local system by the Markovian dynamics of a larger system.

Definition 3 (pseudomode description).-An environment described by $M$ pseudomodes with parameters $\left\{\left(\omega_{i}, g_{i}, \kappa_{i} \geq 0\right): i \in\{1,2 \ldots M\}\right\}$ has an associated Hilbert space $\mathcal{H}_{\text {aux }}$ of $M$ bosonic modes with annihilation operators $a_{0}, a_{1}, \ldots, a_{M-1}$. For a local system with Hilbert space $\mathcal{H}_{S}=\mathbb{C}^{d}$, time-dependent Hamiltonian $H_{S}(t) \in \mathfrak{R}\left(\mathbb{C}^{d}\right)$, interacting with the environment through the operator $L \in \mathfrak{L}\left(\mathbb{C}^{d}\right)$ with the initial system-environment state being $|\sigma\rangle \otimes|\mathrm{vac}\rangle$, its reduced state at time $t$ is given by $\rho(t)=\operatorname{Tr}_{\text {aux }}[R(t)]$, where $R(t)$ satisfies

$$
\begin{aligned}
\dot{R}(t)= & i[\hat{H}(t), R(t)] \\
& +\sum_{i=0}^{M-1} \frac{\kappa_{i}}{2}\left[2 a_{i} R(t) a_{i}^{\dagger}-\left\{a_{i}^{\dagger} a_{i}, R(t)\right\}\right],
\end{aligned}
$$

with

$$
\hat{H}(t)=H_{S}(t)+\sum_{i=0}^{M-1} \omega_{i} a_{i}^{\dagger} a_{i}+\sum_{i=1}^{M} g_{i}\left(a_{i} L^{\dagger}+a_{i}^{\dagger} L\right),
$$

and $R(0)=|\sigma\rangle\langle\sigma| \otimes(|0\rangle\langle 0|)^{\otimes M}$.

An environment described by $M$ pseudomodes with parameters $\left\{\left(\omega_{i}, g_{i}, \kappa_{i} \geq 0\right): i \in\{1,2 \ldots M\}\right\}$ corresponds to a coupling function $\hat{v}$ which satisfies [48]

$$
|\hat{v}(\omega)|^{2}=\sum_{i=1}^{M} \frac{\kappa_{i}}{2 \pi} \frac{g_{i}^{2}}{\left(\omega-\omega_{i}\right)^{2}+\kappa_{i}^{2} / 4} .
$$

In practice, to obtain a pseudomode description that approximates a given coupling function $v,|v|^{2}$ is approximated by a sum of Lorentzians within a sufficiently large but finite frequency window, with each Lorentzian corresponding to an independent pseudomode. Our next result, proved in Supplemental Material [61], shows that this procedure is guaranteed to converge. The convergence rate of the pseudomode approximation will, in general, depend on the details of the coupling function $v$-in particular, on the growth of its derivative $v^{\prime}(\omega)$ with $\omega$, as well as on the falloff of the cutoff error $\varepsilon\left(\omega_{c}, t\right)$, introduced in Theorem 1, with the cutoff frequency. For typical coupling functions, $\left|v^{\prime}(\omega)\right|=$ $O(\operatorname{poly}(\omega))$ and $\varepsilon\left(\omega_{c}, t\right)=O\left(\exp (O(t)) \operatorname{poly}\left(\omega_{c}^{-1}\right)\right)$. Under these assumptions, we show that the error incurred in the pseudomode approximation decreases polynomially with the number of pseudomodes.

Theorem 2 (pseudomode approximation).--Suppose $v \in C_{b}^{\infty}(\mathbb{R}) \cap \mathcal{S}^{\prime}(\mathbb{R})$ is a coupling function such that assumptions 1 and 2 are satisfied and let $\rho(t)$ be the reduced state of the local system after evolving an initial state $|\sigma\rangle \otimes$ $|v a c\rangle$ using the Hamiltonian in Eq. (1). Then, there exists a pseudomode description of the environment (Definition 1) with $M$ bosonic modes which provides an approximation $\hat{\rho}(t)$ to the reduced state of the local system such that $\|\rho(t)-\hat{\rho}(t)\|_{\text {tr }} \rightarrow 0$ as $M \rightarrow \infty$. Furthermore, if $\left|v^{\prime}(\omega)\right|=O(\operatorname{poly}(\omega))$ and the cutoff error $\varepsilon\left(\omega_{c}, t\right)=$ $O\left(\exp (O(t)) \operatorname{poly}\left(\omega_{c}^{-1}\right)\right)$, then there exists a pseudomode description of the non-Markovian system with $M$ bosonic modes such that the trace-norm error in approximating the reduced local system state at time $t$ scales as $O\left(\exp (O(t)) \operatorname{poly}\left(M^{-1}\right)\right)$.

Finally, we consider the star-to-chain transformation, which maps the non-Markovian environment to a 1D bosonic tight-binding model with the local system effectively coupled to the first bosonic mode.

Definition 4 (chain description).-An environment described by a chain of $M$ bosonic modes with parameters $\left\{\left(\omega_{i}, g_{i}\right): i \in\{0,1,2,3 \ldots M-1\}\right\}$ has an associated Hilbert space $\mathcal{H}_{\text {aux }}$ of $M$ bosonic modes with annihilation operators $a_{0}, a_{1}, \ldots, a_{M-1}$, For a local system with Hilbert space $\mathcal{H}_{S}=\mathbb{C}^{d}$, time-dependent Hamiltonian $H_{S}(t) \in \mathfrak{L}\left(\mathbb{C}^{d}\right)$ 
interacting with the environment through the operator $L \in \mathfrak{L}\left(\mathbb{C}^{d}\right)$ with the initial system-environment state being $|\sigma\rangle \otimes \mid$ vac $\rangle$, its reduced state at time $t$ is given by $\rho(t)=\operatorname{Tr}_{\text {aux }}\left[\hat{U}(t, 0)|\sigma\rangle\langle\sigma| \otimes(|0\rangle\langle 0|)^{\otimes M} \hat{U}(0, t)\right]$, where $\hat{U}\left(\tau_{1}, \tau_{2}\right)$ is the propagator corresponding to the Hamiltonian

$$
\hat{H}(t)=H_{S}(t)+g_{0}\left(L^{\dagger} a_{0}+a_{0}^{\dagger} L\right)+H_{E},
$$

with

$$
H_{E}=\sum_{i=0}^{M-1} \omega_{i} a_{i}^{\dagger} a_{i}+\sum_{i=1}^{M-2} g_{i}\left(a_{i} a_{i+1}^{\dagger}+a_{i+1} a_{i}^{\dagger}\right) .
$$

A chain transformation of the environment can be explicitly constructed by using the Lanczos iteration-this proceeds by first introducing a frequency cutoff $\omega_{c}$, and then starting from the mode $a_{0} \propto \int_{-\omega_{c}}^{\omega_{c}} v(\omega) a_{\omega} d \omega$, applying the Lanczos iteration with respect to the environment Hamiltonian. This yields the parameters $\left\{\left(\omega_{i}, g_{i}\right): i \in\right.$ $\{0,1,2 \ldots M-1\}\}$ for the chain description of the environment. By exploiting the previously established connection between the star-to-chain transformation and orthogonal polynomials $[33,70]$, we show (Supplemental Material [61]) that, under Assumptions 1 and 2, the star-tochain transformation will converge for sufficiently large number of modes and that the convergence rate is polynomial in the number of modes.

Theorem 3 (star-to-chain transformation).- Suppose $v \in C_{b}^{\infty}(\mathbb{R}) \cap \mathcal{S}^{\prime}(\mathbb{R})$ is a coupling function such that Assumptions 1 and 2 are satisfied and let $\rho(t)$ be the reduced state of the local system after evolving an initial state $|\sigma\rangle \otimes \mid$ vac $\rangle$ using the Hamiltonian in Eq. (1). Then, there exists a chain description of the environment with $M$ modes (Definition 4) that provides an approximation $\hat{\rho}(t)$ to the reduced state of the local system such that $\|\rho(t)-\hat{\rho}(t)\|_{\text {tr }} \rightarrow 0$ as $M \rightarrow \infty$. Furthermore, if the cutoff error $\varepsilon\left(\omega_{c}, t\right)=O\left(\exp (O(t))\right.$ poly $\left.\left(\omega_{c}^{-1}\right)\right)$, then the tracenorm error in approximating the reduced local system state at time $t$ scales as $O\left(\exp (O(t)) \operatorname{poly}\left(M^{-1}\right)\right)$.

In conclusion, our work provides a rigorous analysis of Markovian dilations to non-Markovian open quantum systems. We show that the finite-time dynamics of a wide class of non-Markovian quantum systems can always be well approximated by a larger Markovian system, and we also provide theoretical scalings of how much larger the effective Markovian system is. Several questions of interest to open quantum system theory are left open in this work. One of the questions that we leave unanswered is to study the class of coupling functions, with possibly distributional kernels, for which the resulting system-environment dynamics is well defined. A rigorous study of this problem would be relevant to advancing the mathematical understanding of non-Markovian open quantum system models, and could lead to a general proof of Assumption 2. Another direction to pursue would be to improve the exponential dependence of the error estimates on time $t$, or to identify settings in which these error estimates are tight.

We thank Martin Plenio, Mischa Woods, Christiane Koch, and Archak Purkayastha for pointing us to relevant literature on this topic. R. T. acknowledges Max Planck Harvard research center for support from the quantum optics (MPHQ) postdoctoral fellowship. We acknowledge support from the ERC Advanced Grant QUENOCOBA under the EU Horizon 2020 program (Grant No. 742102) and from the Deutsche Forschungsgemeinschaft (DFG, German Research Foundation) under Project No. 414325145 in the framework of the Austrian Science Fund (FWF): SFB F7104.

*rahul.trivedi@mpq.mpg.de

[1] H.-P. Breuer, F. Petruccione et al., The Theory of Open Quantum Systems (Oxford University Press on Demand, New York, 2002).

[2] R. Finsterhölzl, M. Katzer, and A. Carmele, Phys. Rev. B 102, 174309 (2020).

[3] A. W. Chin, J. Prior, S. F. Huelga, and M. B. Plenio, Phys. Rev. Lett. 107, 160601 (2011).

[4] S. Groeblacher, A. Trubarov, N. Prigge, G. Cole, M. Aspelmeyer, and J. Eisert, Nat. Commun. 6, 7606 (2015).

[5] I. de Vega, D. Porras, and J. Ignacio Cirac, Phys. Rev. Lett. 101, 260404 (2008).

[6] G. Calajó, Y.-L. L. Fang, H. U. Baranger, and F. Ciccarello, Phys. Rev. Lett. 122, 073601 (2019).

[7] G. Andersson, B. Suri, L. Guo, T. Aref, and P. Delsing, Nat. Phys. 15, 1123 (2019).

[8] A. González-Tudela, C. S. Muñoz, and J. I. Cirac, Phys. Rev. Lett. 122, 203603 (2019).

[9] T. Aref, P. Delsing, M. K. Ekström, A. F. Kockum, M. V. Gustafsson, G. Johansson, P. J. Leek, E. Magnusson, and R. Manenti, in Superconducting Devices in Quantum Optics (Springer, New York, 2016), pp. 217-244.

[10] L. Leonforte, A. Carollo, and F. Ciccarello, Phys. Rev. Lett. 126, 063601 (2021).

[11] A. Ishizaki and Y. Tanimura, J. Phys. Soc. Jpn. 74, 3131 (2005).

[12] A. Chin, S. F. Huelga, and M. B. Plenio, Phil. Trans. R. Soc. A 370, 3638 (2012).

[13] A. Ivanov and H.-P. Breuer, Phys. Rev. A 92, 032113 (2015).

[14] F. Caycedo-Soler, A. Mattioni, J. Lim, T. Renger, S. Huelga, and M. Plenio, arXiv:2106.14286.

[15] M. Xu, Y. Yan, Y. Liu, and Q. Shi, J. Chem. Phys. 148, 164101 (2018).

[16] A. Smirne and B. Vacchini, Phys. Rev. A 82, 022110 (2010).

[17] A. Pereverzev and E. R. Bittner, J. Chem. Phys. 125, 104906 (2006).

[18] L. Kidon, E. Y. Wilner, and E. Rabani, J. Chem. Phys. 143, 234110 (2015). 
[19] B. Vacchini and H.-P. Breuer, Phys. Rev. A 81, 042103 (2010).

[20] M. Schröder, M. Schreiber, and U. Kleinekathöfer, J. Chem. Phys. 126, 114102 (2007).

[21] C. Timm, Phys. Rev. B 83, 115416 (2011).

[22] S. Mukamel, I. Oppenheim, and J. Ross, Phys. Rev. A 17, 1988 (1978).

[23] S. Albeverio, L. Cattaneo, S. Mazzucchi, and L. Di Persio, J. Math. Phys. (N.Y.) 48, 102109 (2007).

[24] D. E. Makarov and N. Makri, Chem. Phys. Lett. 221, 482 (1994).

[25] C. M. Smith and A. O. Caldeira, Phys. Rev. A 36, 3509 (1987).

[26] S. Whalen, A. Grimsmo, and H. Carmichael, Quantum Sci. Technol. 2, 044008 (2017).

[27] A. L. Grimsmo, Phys. Rev. Lett. 115, 060402 (2015).

[28] M. R. Jørgensen and F. A. Pollock, Phys. Rev. Lett. 123, 240602 (2019).

[29] J. del Pino, F. A. Y. N. Schröder, A. W. Chin, J. Feist, and F. J. Garcia-Vidal, Phys. Rev. Lett. 121, 227401 (2018).

[30] A. Strathearn, P. Kirton, D. Kilda, J. Keeling, and B. W. Lovett, Nat. Commun. 9, 3322 (2018).

[31] R. Rosenbach, J. Cerrillo, S. F. Huelga, J. Cao, and M. B. Plenio, New J. Phys. 18, 023035 (2016).

[32] J. Huh, S. Mostame, T. Fujita, M.-H. Yung, and A. AspuruGuzik, New J. Phys. 16, 123008 (2014).

[33] A. W. Chin, Á. Rivas, S. F. Huelga, and M. B. Plenio, J. Math. Phys. (N.Y.) 51, 092109 (2010).

[34] M. Woods, R. Groux, A. Chin, S. F. Huelga, and M. B. Plenio, J. Math. Phys. (N.Y.) 55, 032101 (2014).

[35] G. Vidal, Phys. Rev. Lett. 93, 040502 (2004).

[36] A. J. Daley, C. Kollath, U. Schollwöck, and G. Vidal, J. Stat. Mech. (2004) P04005.

[37] M.-C. Banuls, M. B. Hastings, F. Verstraete, and J. I. Cirac, Phys. Rev. Lett. 102, 240603 (2009).

[38] A. J. Daley, J. M. Taylor, S. Diehl, M. Baranov, and P. Zoller, Phys. Rev. Lett. 102, 040402 (2009).

[39] F. Verstraete, J. J. Garcia-Ripoll, and J. I. Cirac, Phys. Rev. Lett. 93, 207204 (2004).

[40] D. W. Berry, A. M. Childs, R. Cleve, R. Kothari, and R. D. Somma, in Proceedings of the Forty-Sixth Annual ACM Symposium on Theory of Computing (Association for Computing Machinery (ACM), New York, 2014), pp. 283-292.

[41] G. H. Low and I. L. Chuang, Quantum 3, 163 (2019).

[42] D. W. Berry, A. M. Childs, and R. Kothari, in Proceedings of the 2015 IEEE 56th Annual Symposium on Foundations of Computer Science (IEEE, New York, 2015), pp. 792-809.

[43] P. Zanardi, J. Marshall, and L. Campos Venuti, Phys. Rev. A 93, 022312 (2016).

[44] A. Chenu, M. Beau, J. Cao, and A. del Campo, Phys. Rev. Lett. 118, 140403 (2017).

[45] R. Cleve and C. Wang, arXiv:1612.09512.

[46] A. A. Budini, Phys. Rev. A 63, 012106 (2000).

[47] N. Megier, W. T. Strunz, C. Viviescas, and K. Luoma, Phys. Rev. Lett. 120, 150402 (2018).

[48] D. Tamascelli, A. Smirne, S. F. Huelga, and M. B. Plenio, Phys. Rev. Lett. 120, 030402 (2018).

[49] G. Pleasance, B. M. Garraway, and F. Petruccione, Phys. Rev. Research 2, 043058 (2020).
[50] L. Mazzola, S. Maniscalco, J. Piilo, K.-A. Suominen, and B. M. Garraway, Phys. Rev. A 80, 012104 (2009).

[51] B. J. Dalton, S. M. Barnett, and B. Garraway, Phys. Rev. A 64, 053813 (2001).

[52] B. Garraway and B. Dalton, J. Phys. B 39, S767 (2006).

[53] B. Dalton, S. M. Barnett, and B. Garraway, in Coherence and Quantum Optics VIII: Proceedings of the Eighth Rochester Conference on Coherence and Quantum Optics, University of Rochester, 2001 (Springer Science \& Business Media, New York, Boston, 2012), p. 495.

[54] M. P. Woods, M. Cramer, and M. B. Plenio, Phys. Rev. Lett. 115, 130401 (2015).

[55] G. Gualdi and C. P. Koch, Phys. Rev. A 88, 022122 (2013).

[56] F. Mascherpa, A. Smirne, S. F. Huelga, and M. B. Plenio, Phys. Rev. Lett. 118, 100401 (2017).

[57] J. Eisert and D. Gross, Phys. Rev. Lett. 102, 240501 (2009).

[58] S. Fan, Ş. E. Kocabaş, and J.-T. Shen, Phys. Rev. A 82, 063821 (2010).

[59] A map $v: \mathbb{R} \rightarrow \mathbb{C}$ is in $L^{2}(\mathbb{R})$ if $v$ is square integrable with respect to the Lebesgue measure.

[60] A map $v: \mathbb{R} \rightarrow \mathbb{C}$ is in $C_{b}^{\infty}(\mathbb{R})$ if $v$ is smooth and bounded. For $v \in C_{b}^{\infty}(\mathbb{R}),\|v\|_{\infty}=\sup _{\omega \in \mathbb{R}}|v(\omega)|$. We will denote the space of tempered distributions over $\mathbb{R}$ by $\mathcal{S}^{\prime}(\mathbb{R})-C_{b}^{\infty}(\mathbb{R}) \cap \mathcal{S}^{\prime}(\mathbb{R})$ is the space of tempered distributions that correspond to multiplication with bounded and smooth functions.

[61] See Supplemental Material at http://link.aps.org/ supplemental/10.1103/PhysRevLett.127.250404 for detailed proofs of the lemmas, propositions and, theorems in the Letter, together with an extension of Theorem 1 for initially excited environment states, which includes Refs. [62-64].

[62] R. Trivedi, K. Fischer, S. Xu, S. Fan, and J. Vuckovic, Phys. Rev. B 98, 144112 (2018).

[63] T. Caneva, M. T. Manzoni, T. Shi, J. S. Douglas, J. I. Cirac, and D. E. Chang, New J. Phys. 17, 113001 (2015).

[64] R. McClarren, Computational Nuclear Engineering and Radiological Science Using PYTHON, edited by R.G. McClarren (Academic Press, Cambridge, 2018), p. 287.

[65] A map $f:[a, b]^{2} \rightarrow \mathbb{C}$ will be called symmetric if $\forall s_{1}$, $s_{2} \in[a, b]: f\left(s_{1}, s_{2}\right)=f^{*}\left(s_{2}, s_{1}\right)$. We will denote by $A C_{\text {sym }}\left([a, b]^{2}\right)$ the space of symmetric, continuous functions $f:[a, b]^{2} \rightarrow \mathbb{C}$ which satisfy the following two conditions: (i) $f$ is absolutely continuous in either of its arguments, i.e., $\forall y \in[a, b]$, the map $\varphi_{y}:[a, b] \rightarrow \mathbb{C}$ defined by $\varphi_{y}(x)=$ $f(x, y)$ is absolutely continuous. (ii) For $y \in_{a . e .}[a, b]$, $\partial_{y} f(x, y)$ exists $\forall x \in[a, b]$ and the map $\varphi_{y}^{d}:[a, b] \rightarrow \mathbb{C}$ defined by $\varphi_{y}^{d}(x)=\partial_{y} f(x, y)$ is absolutely continuous. We note that the partial derivatives of $f$ with respect to either and both of its arguments, $\partial_{x} f, \partial_{y} f, \partial_{x, y}^{2} f$, exists almost everywhere on $[a, b]^{2}$. Finally, we define a norm $\|\cdot\|_{[a, b]^{2}}: A C_{\text {sym }}\left([a, b]^{2}\right) \rightarrow \mathbb{R}^{+}$such that $\forall f \in$ $A C_{\text {sym }}\left([a, b]^{2}\right)$

$$
\begin{aligned}
\|f\|_{[a, b]^{2}}^{\mathcal{S}}= & \sup _{x, y \in[a, b]}|f(x, y)| \\
& \left.+(b-a) \underset{x, y \in[a, b]}{\operatorname{esssup}}\left|\partial_{x} f(x, y)\right|+\underset{x, y \in[a, b]}{\operatorname{esssup}}\left|\partial_{y} f(x, y)\right|\right) \\
& +(b-a)^{2} \operatorname{esssup}_{x, y \in[a, b]}\left|\partial_{x, y}^{2} f(x, y)\right| .
\end{aligned}
$$


A symmetric map $K$ is also positive if

$\forall g \in C^{0}([a, b]): \int_{s_{1}, s_{2}=a}^{b} K\left(s_{1}, s_{2}\right) g^{*}\left(s_{1}\right) g\left(s_{2}\right) d s_{1} d s_{2} \geq 0$.

We define $\quad A C_{\text {sym }}^{\geq 0}\left([a, b]^{2}\right)=\left\{f \in A C_{\text {sym }}\left([a, b]^{2}\right) \mid f\right.$ is positive.\}.

[66] A. Barut and J. Kraus, Found. Phys. 13, 189 (1983).

[67] B. M. Garraway, Phys. Rev. A 55, 2290 (1997).
[68] D. Cilluffo, A. Carollo, S. Lorenzo, J. A. Gross, G. M. Palma, and F. Ciccarello, Phys. Rev. Research 2, 043070 (2020).

[69] For a Hilbert space $\mathcal{H}, \mathfrak{Q}(\mathcal{H})$ is the set of operators $T: \mathcal{H} \rightarrow \mathcal{H}$ which are bounded. For $T \in \mathfrak{Q}\left(\mathcal{H}_{S}\right),\|\cdot\|$ is the usual operator norm defined by $\|T\|=$ $\sup _{|\psi\rangle \in \mathcal{H}_{S} \backslash\{0\}} \| T|\psi\rangle\|/\||\psi\rangle \|$.

[70] I. de Vega, U. Schollwöck, and F. A. Wolf, Phys. Rev. B 92, 155126 (2015). 